\title{
Stage I Osteosarcoma AJCC v7
}

National Cancer Institute

\section{Source}

National Cancer Institute. Stage I Osteosarcoma A/CC v7. NCI Thesaurus. Code C6709.

Stage I includes: IA (T1, N0, M0, G1, G2, GX); IB (T2, N0, M0, G1, G2, GX); (T3, N0, M0,

G1, G2, GX). T1: Tumor $8 \mathrm{~cm}$ or less in greatest dimension. T2: Tumor more than $8 \mathrm{~cm}$ in greatest dimension. T3: Discontinuous tumors in the primary bone site. N0: No regional lymph node metastasis. M0: No distant metastasis. G1: Well differentiated-low grade.

G2: Moderately differentiated-low grade. GX: Grade cannot be assessed. (AJCC 7th ed.) 